\title{
QRS Fragmentation as a Prognostic Test in Acute Coronary Syndrome
}

\author{
Tarek M. Abdelrahman \\ Cardiology Department, Minia University Hospital, Minia, Egypt \\ Email: tarekabdelrahman211@yahoo.com
}

Received February 26, 2013; revised March 26, 2013; accepted April 4, 2013

Copyright (C) 2013 Tarek M. Abdelrahman. This is an open access article distributed under the Creative Commons Attribution License, which permits unrestricted use, distribution, and reproduction in any medium, provided the original work is properly cited.

\begin{abstract}
Background-Rationale of the Study: Among several invasive and non-invasive tests for risk stratification of acute coronary syndromes (ACS), fewer markers can be utilized in clinical practice. Our rationale is to validate use of QRS-fragmentation as a promising bed-side test for assessment of prognosis in those patients. Methods and Results: Collection of two-hundred and twenty patients with ACS was done during two years (from January 2011 till January 2013). Significant organic vaLVe disease and QRS duration $\geq 120 \mathrm{~ms}$ as well as patients with permanent pacemakers were excluded. Patients were subjected to full clinical examination, ECG and Echocardiography in the first day of admission followed by diagnostic coronary angiography before discharge and a nuclear study was done for Randomized sample from each group. 12-leads ECG revealed fragmentation of QRS in 74 patients and 146 patients with no QRS fragmentation. Localization of the infarct site revealed NS difference between percentages in both groups. Echocardiography revealed a significant deterioration of LV functions in group-A than group-B. Also, MR jet area was significantly higher in group-A. Coronary angiography revealed severer lesions in group-A more than group-B. Nuclear study revealed higher percentages of irreversible scars in group-A (30\%) and higher reversibility in group-B (80\%). In-hospital Occurrence of complications from ACS revealed a significant higher incidence of MACE in group-A. Conclusion: Presence of fragmented QRS in surface ECG during ACS represents myocardial scar or fibrosis and reflects the severity of coronary lesions and a correlation between fQRS and depression of LV function is established. Indeed, occurrence of MACE is suspected.
\end{abstract}

Keywords: Fragmented QRS (fQRS); Acute Coronary Syndrome (ACS); Major Adverse Cardiac Events (MACE)

\section{Introduction}

Observation of Slurring and changes in the morphology of the QRS complex has been investigated since 1960's. Flowers et al. discovered presence of fragmented QRS (fQRS) complexes among patients with prior myocardial infarction and patients with either right or left ventricular (LV) enlargement and reported it as high-frequency components [1]. Friedman et al. described this fragmentation as persistent changes in Purkinje fibers and myocardial fibrosis which may cause slow and inhomogeneous myocardial activation after analyzing canine heart with induced myocardial infarction [2].

Varriale and Chryssos suggested that RSR' complex

\footnotetext{
"Funding Sources: All the study fees were sponsored by the governmental policies to support the research work in my university according to the established financial protocols.

Disclosures: None.

Ethics Approval: All patients wrote a written approval to be included in the research work.
}

unrelated to right bundle branch block (RBBB) or left bundle branch block (LBBB), could be associated with impaired depolarization within tissue surrounding the myocardial scar [3]. Bayes de Luna suggested that abnormalities of the second half of the QRS complex (terminal slurring, sometimes with R' in lead V1) during MI might represent necrosis in late depolarized basal zones [4].

Contemporary definition of the fQRS was defined by Das et al. in 2006, as presence of an additional $\mathrm{R}$ wave (R') or notching in the nadir of the S wave, or the presence of $>1 R^{\prime}$ in 2 contiguous leads, corresponding to a major coronary artery territory on the resting 12-lead ECG with filter range $0.16-100 \mathrm{~Hz}, \mathrm{AC}$ filter $60 \mathrm{~Hz}$, paper speed $25 \mathrm{~mm} / \mathrm{s}$ and $10 \mathrm{~mm} / \mathrm{mV}$ [5].

It has been shown in some studies that abnormality within the QRS complex can represent conduction disturbance and myocardial scar. Injured tissue around an infarct scar resulted in the RSR' pattern of the QRS com- 
plex. However, the diagnostic and prognostic values of these subtle abnormalities within the QRS complex were not clarified in prior studies $[5,6]$.

In 2006, Das et al. proved that, fragmented QRS complex in patients with coronary artery disease (CAD) was associated with myocardial conduction block due to myocardial scar detected by myocardial single photon emission tomography (SPECT). fQRS improved identification of prior myocardial infarction in patients who are being evaluated for CAD [5].

As fQRS represents prior occurrence of myocardial infarction, further studies are directed to its role in identifying the risk of subsequent occurrence of ischemic events, its correlation to ventricular dysfunction and occurrence of congestive heart failure. Das et al. demonstrated that $\mathrm{fQRS}$ is an independent predictor of cardiac events in patients with CAD [6]. Other studies confirmed a relation between $\mathrm{fQRS}$ and LV dysfunction $[7,8]$.

Myocardial scar is also a substrate for reentrant ventricular tachyarrhythmia. Signal averaged electrocardiogram (SAECG) reveals the presence of late potential that indicates low-amplitude high-frequency potentials outside the terminal QRS complex. Abnormal late potential represents a slow conduction zone with damaged myocardium around the fibrosis of healed myocardial infarction [9]. The presence of late potential has been used for risk stratification of sudden cardiac death or lethal arrhythmic events [10].

As well as SAECG, fQRS also can reflect intra-cardiac conduction abnormality and will represent a substrate for ventricular arrhythmia. There has been only one study in which the correlation between fQRS and late potential detected by SAECG was investigated. This study demonstrated that the existence of fQRS appeared independently from the existence of late potential in patients with Brugada syndrome, but it is still unknown whether there is a correlation between fQRS and late potential in other diseases such as CAD and various cardiomyopathies [11].

\section{2. fQRS in Various Diseases}

\subsection{Coronary Artery Disease (CAD)}

It was demonstrated that the fragmentation of QRS complexes represent myocardial scar in patients with suspected or known CAD in two major studies, one for narrow (QRS duration $<120 \mathrm{~ms}$ ) [5] and the other for wide (QRS duration $\geq 120 \mathrm{~ms}$ ) [12]. Sensitivity, specificity, and the negative predictive value for myocardial scar were $86 \%, 89 \%$, and $93 \%$, respectively, for the fQRS in the first study. And the second study was on 879 patients showed a sensitivity, specificity, positive predictive value and negative predictive value of $\mathrm{f}-\mathrm{wQRS}$ for myocardial scar were $87 \%, 93 \%, 92 \%$ and $88 \%$, respectively [12].

Gardner et al. proposed that fQRS complex in infarc- ted canine heart is caused by slow and inhomogeneous activation related to healed myocardial scar rather than changes in transmembrane resting or action potentials [13]. In the analysis of data from an epicardial and endocardial mapping from the patients undergoing LV aneurysm incision, Wiener et al. [14] demonstrated that, the fragmented electrical activity was present in all patients with LV aneurysms. Indeed he found that, patients with ventricular tachycardia had fragmented electrical activity than patients without ventricular tachycardia. The surgical excision of the area, which demonstrated fragmented activity, was associated with better survival and freedom from ventricular arrhythmia. Wiener et al. proposed that endocardial electrical activity mapping and detection of fragmented activity was a useful tool for surgically guided therapy for ventricular aneurysm and ventricular tachycardia [15].

\subsection{Non-Coronary Artery Disease (Non-CAD)}

1) Dilated cardiomyopathy (DCM): In patients with DCM, QRS fragmentation has been recorded during wavelet ECG analysis recorded from 6 unipolar left precordial leads, using a high-precision amplifier. When compared with the control subjects with no heart disease, these wavelets predicted frequent premature ventricular complexes and SCD [16]. In DCM, fQRS signifies myocardial scar defined by gadolinium delayed enhancement on cardiac magnetic resonance imaging. fQRS also signifies myocardial scar in non-CAD patients [17].

2) Cardiac sarcoidosis: Homsi et al. demonstrated that fQRS is a risk marker of cardiac sarcoidosis. The sensitivity and specificity of fQRS for detecting abnormal gadolinium-delayed enhancement images in cardiac magnetic resonance imaging were $100 \% \& 80 \%$, respectively [18].

3) $A R V D / C$ : fQRS has been identified as a risk marker of ARVD/C by Peters et al. They studied the value of QRS fragmentation in a standard 12-lead ECG in 360 patients with ARVD/C and compared its presence with the detection of the epsilon wave in highly amplified right precordial and modified limb leads in a subgroup of 207 patients. Other ECG signs of ARVD/C include QRS prolongation, prolonged S-wave upstroke, terminal activation delay, and epsilon potentials. Most of these signs are incorporated in typical fQRS. fQRS will probably simplify the ECG diagnosis of ARVD/C in patients with a high probability of the disease [19].

\subsection{Electrical Diseases}

1) Brugada syndrome: is characterized by cove-type ST elevation in right precordial leads and episodes of ventricular tachyarrhythmia. Patients with Brugada syndrome often had fQRS and it was more frequently ob- 
served in the VF group [20]. Recently, the PRELUDE study has shown that fQRS was useful for identify candidates for a prophylactic ICD implantation in patients with Brugada syndrome [21].

2) Acquired long QT syndrome: (ALQTS) is a disease due to secondary repolarization abnormality. Repolarization abnormality, such as prolongation of QT interval and prolongation of the peak to end of $\mathrm{T}$ wave represented transmural or intraventricular dispersion of repolarization, and was associated with occurrence of tosades de pointes [22]. It was found that fQRS was present in a large percentage of ALQTS patients with syncope/torsades de pointes $(81 \%)$. Although onset of torsades de pointes is triggered activity due to early after depolarization, myocardial scar that appears as fQRS could be a substrate for subsequent reentrant arrhythmia [23].

\subsection{Congenital and Rheumatic HD}

In adult patients with repaired tetralogy of Fallot, fQRS predicted ventricular fibrosis detected by late Ga-MRI. Laterality of fQRS was associated with operative scar of the RV, and patients with fQRS had larger RV volume and lower RV EF. fQRS was closely associated with more extensive RV fibrosis and dysfunction [24]. fQRS was frequent in patients with mitral stenosis caused by rheumatic fever. Rheumatic fever induces inflammation and degeneration of the cardiac valves and also injury of the myocardium. fQRS was associated with low EF, pulmonary hypertension, poor NYHA functional class, and decreased mitral vaLVe area [7].

\subsection{Miscellaneous Diseases}

There has been an interest in fQRS as a screening tool in a systemic disease with cardiac involvement. Kadi et al. assessed the frequency of fQRS complexes in patients with rheumatoid arthritis (RA) and in control group of patients with fibromyalgia rheumatica. Patients with BBB and cardiovascular disease were excluded. Fragmented QRS were more often found among the patients with RA than a control group [25].

\section{Patients and Methods}

In Cardiology department of Minia university hospital two-hundred and twenty eligible patients with ACS during two years (from January 2011 till January 2013) were enrolled consecutively in this study. Patients with significant organic vaLVe disease and those with any QRS morphology that had a QRS duration $\geq 120 \mathrm{~ms}$ as well as patients with permanent pacemakers were excluded from this study.

All patients-in the first day-were subjected to full clinical examination, ECG using Fukuda Denshi Climax machine. A 12-lead admission ECG was obtained from all patients. All ECGs (filter range $0.5 \mathrm{~Hz}$ to $150 \mathrm{~Hz}, \mathrm{AC}$ filter $60 \mathrm{~Hz}, 25 \mathrm{~mm} / \mathrm{s}, 10 \mathrm{~mm} / \mathrm{mV}$ ) were analyzed by two independent clinicians who were blinded to the study design. The fQRS was defined by the presence of various RSR' patterns (QRS duration $<120 \mathrm{~ms}$ ) with or without $\mathrm{Q}$ wave, which included an additional $\mathrm{R}$ wave ( $\mathrm{R}$ ' prime) or notching of the R wave or S wave, or the presence of more than one $\mathrm{R}$ prime (fragmentation) without typical bundle branch block in two contiguous leads corresponding to a major lead set for major coronary artery territory. The presence of fQRS was detected by inspection of tracings with the naked eye. Analysis of the standard 12-lead ECG was performed without using any magnification. As shown in Figure 1

Echocardiography using a commercially available GE vivid 3 echo machine equipped with $2.5-3.5 \mathrm{MHz}$ transducer to examine for LV volumes including (end-diastolic EDV, end-systolic ESV) through which calculation of LVEF\% is made by Simpson's Biplane method. Also, recording wall motions abnormalities and presence of aneurysm was done. Laboratory analysis of troponin-I and other routine investigations were recorded.

Patients are then divided into two groups (group-A) 74 patients having fQRS in their resting ECG represents $33.6 \%$ and (group-B) were 146 patients having either pathological "q" waves or repolarization changes only with

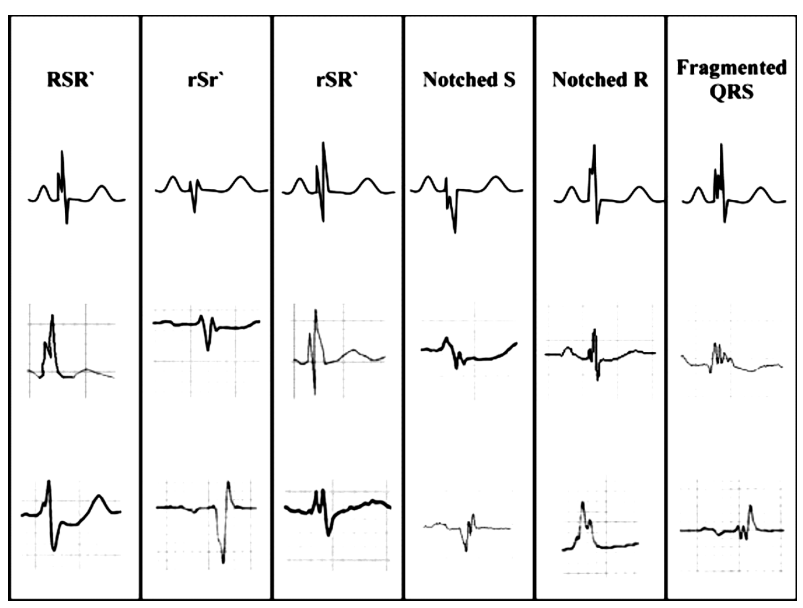

Figure 1. Fragmented QRS (RSR2 pattern and its variants). The different morphologies of fragmented QRS (fQRS), which include various RSR2 patterns, are shown in this figure. Note that if RSR2 patterns are present in right precordial leads (leads V1 and V2) with QRS > 100 msec (incomplete right bundle branch block), or QRS $>120 \mathrm{msec}$ (complete right bundle branch block) and in left precordial lead (RSR2 in lead I, V5, and V6) with QRS > 120 msec (left bundle branch block), they are defined as complete or incomplete bundle branch block and excluded from the definition of fQRS, whereas if the RSR2 pattern is present in the mid-precordial lead or in inferior leads, they are defined as fQRS [6]. 
no fQRS and represents $66.4 \%$.

All cases underwent diagnostic coronary angiography (CA) before discharge using a flat-panel GE-imaging system. All subjects were in the fasting sedated state. CA was performed from the arterial femoral approach after local groin infiltration of $10-20 \mathrm{ml}$ xylocaine $2 \%$ using modified seldinger's technique after injection of $5000 \mathrm{IU}$ of Heparin, 6F JL then JR coronary catheters were used to engage the corresponding arteries. The study was conducted with a General Electric Innova 2000 angiographic unit (GE medical system Milwaukee, WI, USA).

Due to lack of nuclear scan in our hospital, a randomized selection of 20 patients in each group was sent to nuclear study in specialized centers. From group (A): 10 patients having anterior MI (anteroseptal and anterolateral MI were included) and 10 patients having inferior wall MI (inferopsterior and inferolateral MI were included). The same sample was taken from group (B) also as to compare their scan results. As shown in Figure 2.

Clinical characteristics, which consisted of multiple descriptors from each patient's history and physical examination, were collected by physicians from cardiology clinic for each patient before cardiac catheterization and were stored in the database of coronary angiography laboratory at our institution. We recorded the baseline characteristics, which include hypertension, diabetes mellitus, smoking history, family history for coronary artery disease (CAD) and lipid parameters. Patients with significant organic valvular heart disease and BBB (Left $\mathrm{BBB}$, incomplete or complete Right $\mathrm{BBB}$ or duration $\mathrm{QRS}>120 \mathrm{~ms}$ ), and patients with permanent pacemakers were excluded from the study.

MACE were defined as cardiac death, re-infarction, heart failure, cerebrovascular event, sustained ventricular tachycardia or fibrillation. Low cardiac output syndrome was defined as usage of inotropic or intraaortic balloon pump support for more than 30 minutes in the coronary care unit in order to maintain adequate hemodynamics after appropriate adjustment of preload and afterload.

\section{Statistical Methods}

Data were collected and analyzed by Statistical Package for Social Sciences (SPSS) version 13 Data are given as mean (SD) for both numbers and percentages and compared using Chi-square and the Student t test. Pearson's correlation was made for the relations between MACE and myocardial scars in nuclear scan to fQRS positive patients. A probability level of $p$-value $\leq 0.05$ was considered as statistically significant in all tests

\subsection{Results}

Patients characteristics as regard Age, Gender, BMI, Concomitant medical diseases (hypertension, diabetes
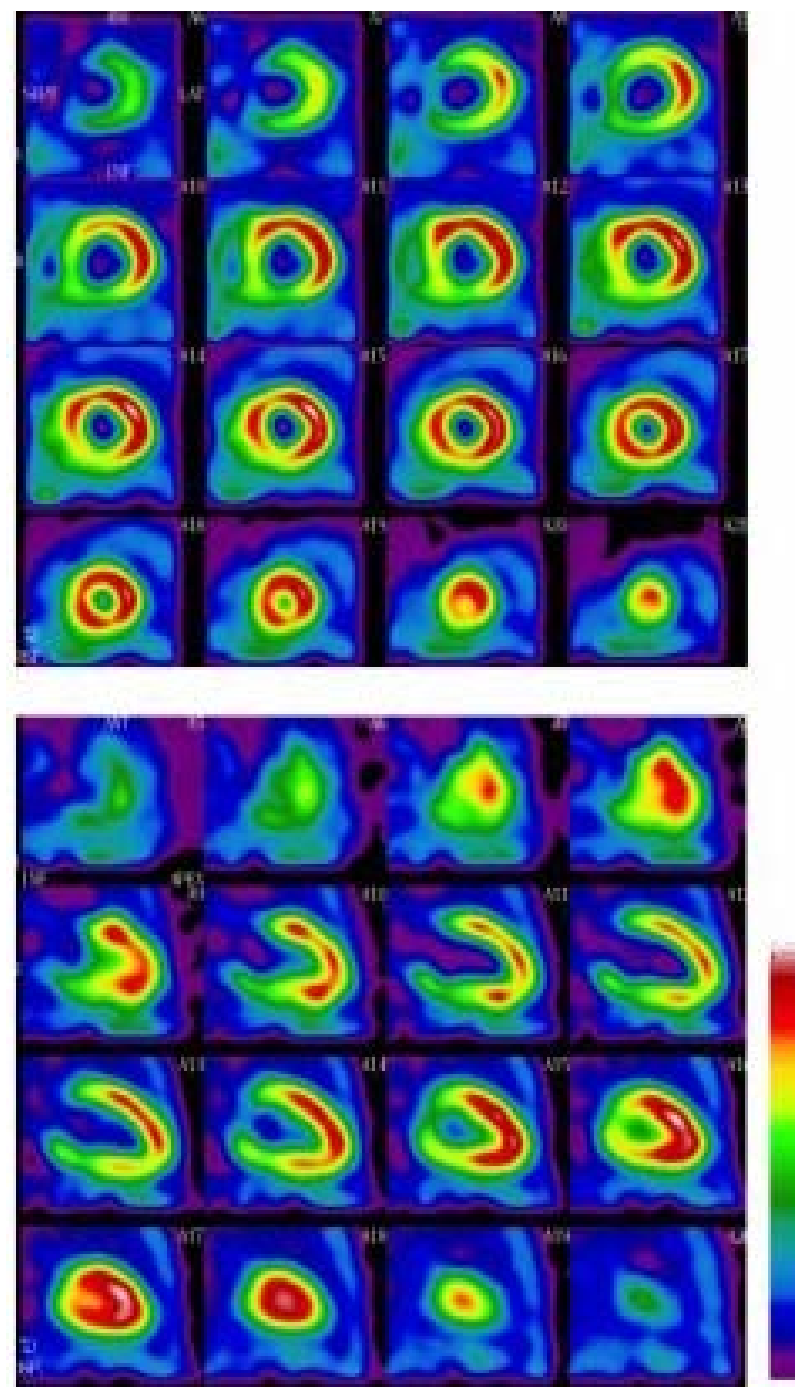

Figure 2. Nuclear imaging (99 m Tc-TF) showed a fixed inferior wall myocardial perfusion defect in patient with recent inferior Myocardial infarction.

mellitus and Hyperlipidemia), Renal functions and medications used inside CCU are shown in Table 1 and revealed no significant statistical differences between both fragmented and non-fragmented groups.

12-leads ECG revealed presence of fragmentation of QRS in 74 patients named as (group-A) and 146 patients with no QRS fragmentation and named as (group-B). Localization of the infarct site in each group revealed presence of anterior MI in 36 patients in group-A represents $(48.6 \%)$ of the total group and 68 patient in group-B (46.5\%), inferior MI were 12 patient in group-A $(16.2 \%)$ and 34 patients in group-B $(23.3 \%)$, anterolateral MI were 9 patients in group-A $(12.3 \%$ and 18 patients $(12.4 \%)$ in group-B, inferolateral MI were 6 patients in group-A $(8.1 \%)$ and 12 patients $(8.3 \%)$ in group$\mathrm{B}$, inferoposterior MI were $6(8.1 \%)$ in group-A versus 8 patients $(5.3 \%)$ in group-B and finally, posterolateral 
Table 1. Baseline characteristics of the study population.

\begin{tabular}{|c|c|c|c|}
\hline \multirow{2}{*}{ Parameter } & \multicolumn{2}{|c|}{ Fragmentation of QRS in admission ECG } & \multirow{2}{*}{ p-Value } \\
\hline & Fragmented QRS Group (A): $n=74$ (33.6\%) & Non-fragmented QRS Group (B): $n=146(66.4 \%)$ & \\
\hline Age (yrs) & $55 \pm 9$ & $56 \pm 8$ & NS \\
\hline Height (cm) & $167 \pm 4$ & $166 \pm 8$ & NS \\
\hline Weight (kg) & $85 \pm 2$ & $84 \pm 6$ & NS \\
\hline BMI $\left(\mathrm{kg} / \mathrm{m}^{2}\right)$ & $33.2 \pm 4$ & $32.8 \pm 8$ & NS \\
\hline Gender male, n (\%) & $61(82 \%)$ & $122(83 \%)$ & NS \\
\hline Hypertension, $\mathrm{n}(\%)$ & $38(51 \%)$ & $77(52 \%)$ & NS \\
\hline Diabetes mellitus, n (\%) & $40(53 \%)$ & $76(52 \%)$ & NS \\
\hline Hyperlipidemia, (n\%) & $52(70 \%)$ & $102(69 \%)$ & NS \\
\hline Serum creatinine $(\mathrm{mg} \%)$ & $1.5 \pm 0.2$ & $1.3 \pm 0.3$ & NS \\
\hline \multicolumn{4}{|l|}{ Medications } \\
\hline ASA, n (\%) & $62(83 \%)$ & $119(81 \%)$ & NS \\
\hline Clopidogrel, n (\%) & $18(24 \%)$ & $32(22 \%)$ & NS \\
\hline ACEI, n (\%) & $33(44 \%)$ & $62(42 \%)$ & NS \\
\hline ARB, n (\%) & $22(29 \%)$ & $46(31 \%)$ & NS \\
\hline B-Blockers, n (\%) & $66(89 \%)$ & $128(87 \%)$ & NS \\
\hline $\mathrm{CCB}, \mathrm{n}(\%)$ & $12(16 \%)$ & $21(14 \%)$ & NS \\
\hline Statin, n (\%) & $28(37 \%)$ & $56(38 \%)$ & NS \\
\hline Diuretics, n (\%) & $8(10.8 \%)$ & $18(12 \%)$ & NS \\
\hline OAD/Insulin, n (\%) & $17(12 \%)$ & $19(13 \%)$ & NS \\
\hline
\end{tabular}

MI were $5(6.7 \%)$ in group-A versus $6(4.2 \%)$ in group-B. As shown in Table 2, Figures 3 and 4. Chi square analysis for descriptive values revealed non-significant differences between both groups at any site.

Echocardiograhic findings in both groups were tabulated and revealed a significant increase in Lv volumes and significant decrease in $\mathrm{EF} \%$ and $\mathrm{FS} \%$ in group-A than group-B. Also, MR jet area was significantly high in group-A which concludes deterioration in LV functions in group-A. As shown in Table 3.

\subsection{Coronary Angiographic Findings}

Semi-quantitative measurement of lesions severity revealed more significant lesions in group-A than in groupB as shown in Table 4.

\subsection{Nuclear Findings}

Twenty patients were randomly selected from each group and detection of irreversible scar tissue and reversible ischemia in every group was calculated. Results revealed a significantly higher percentages of irreversible scars in
Table 2. ECG localization of infarct site.

\begin{tabular}{lccc}
\hline \multicolumn{1}{c}{ Site of MI } & $\begin{array}{c}\text { Group-A } \\
(\mathrm{n}=74)\end{array}$ & $\begin{array}{c}\text { Group-B } \\
(\mathrm{n}=146)\end{array}$ & $\mathrm{p}$ value \\
\hline Anterior MI & $36(48.6 \%)$ & $68(46.5 \%)$ & NS \\
Inferior MI & $12(16.2 \%)$ & $34(23.3 \%)$ & NS \\
Lateral MI & & & \\
$\quad$ anterolateral & $9(12.3 \%)$ & $18(12.4 \%)$ & NS \\
$\quad$ inferolateral & $6(8.1 \%)$ & $12(8.3 \%)$ & \\
Posterior MI & & & \\
inferoposterior & $6(8.1 \%)$ & $8(5.3 \%)$ & NS \\
posterolateral & $5(6.7 \%)$ & $6(4.2 \%)$ & \\
\hline
\end{tabular}

group-A (30\%) and higher percentages of reversibility in group-B (80\%). As shown in Figure 5.

\section{MACE}

In-hospital Occurrence of complications from ACS were studied and revealed a significant $(\mathrm{p}<0.05)$ higher inci- 


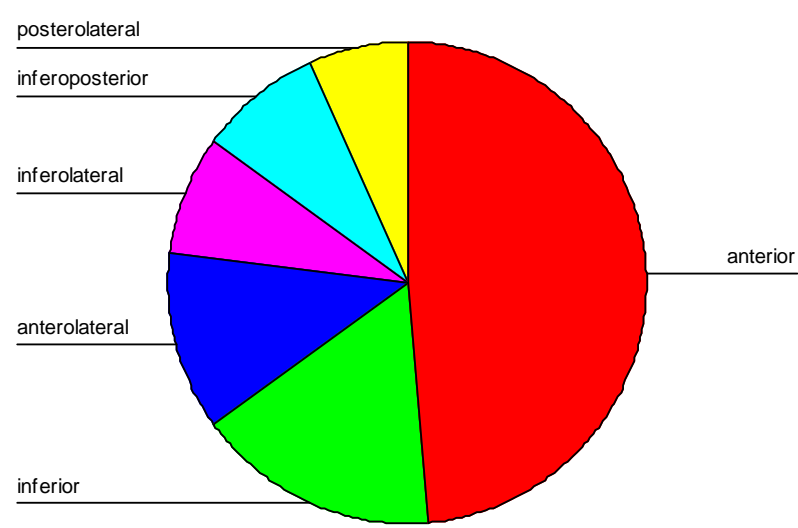

Figure 3. Localization of infarct-site in group-A.

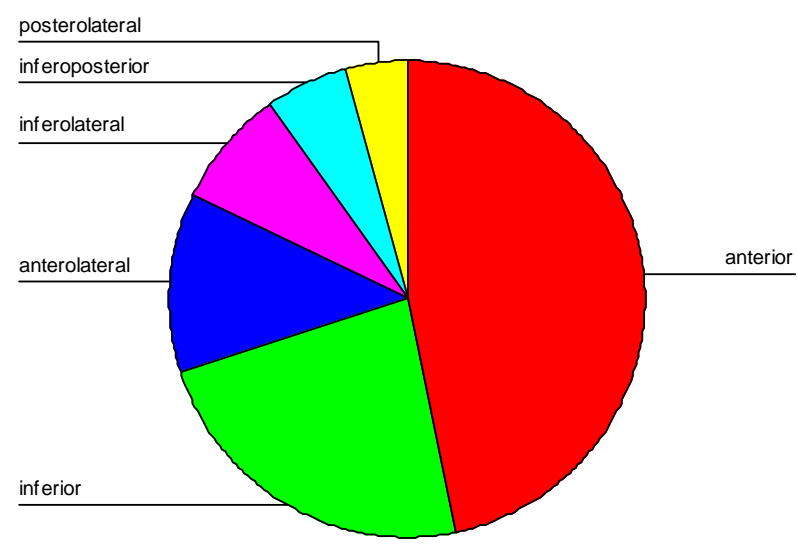

Figure 4. Localization of infarct-site in group-B.

Table 3. Echocardiographic findings.

\begin{tabular}{lccc}
\hline Echocardiograhic Finding & $\begin{array}{c}\text { Group-A } \\
(\mathrm{n}=74)\end{array}$ & $\begin{array}{c}\text { Group-B } \\
(\mathrm{n}=146)\end{array}$ & $\begin{array}{c}\mathrm{p} \\
\text { value }\end{array}$ \\
\hline Lv end diastolic volume (LVEDV) $\mathrm{ml}$ & $131 \pm 18$ & $98 \pm 12$ & 0.001 \\
Lv end systolic volume (LVESV) $\mathrm{ml}$ & $88 \pm 15$ & $65 \pm 5$ & 0.001 \\
Ejection Fraction (EF\%) & $49 \pm 4$ & $61 \pm 4.4$ & 0.001 \\
Fractional shortening (FS\%) & $20 \pm 2.1$ & $27 \pm 2.8$ & 0.001 \\
MR jet area width $\left(\mathrm{cm}^{2}\right)$ & $2.5 \pm 0.4$ & $0.7 \pm 0.2$ & 0.001 \\
\hline
\end{tabular}

Table 4. Semi-quantitative measurement of severity of lesions.

\begin{tabular}{|c|c|c|c|}
\hline \multirow{2}{*}{ Coronary artery } & \multicolumn{2}{|c|}{ Lesion severity } & \multirow{2}{*}{$\mathrm{p}$ value } \\
\hline & $\begin{array}{c}\text { Group-A } \\
(\mathrm{n}=74)\end{array}$ & $\begin{array}{l}\text { Group-B } \\
(\mathrm{n}=146)\end{array}$ & \\
\hline Left Main artery (LM) & $40 \% \pm 10$ & $20 \%$ & 0.001 \\
\hline Left anterior descending A (LAD) & $80 \% \pm 20$ & $40 \% \pm 10$ & 0.001 \\
\hline Diagonal branch artery (DA) & $90 \% \pm 10$ & $50 \% \pm 10$ & 0.001 \\
\hline Left circumflex artery $(\mathrm{LCx})$ & $60 \% \pm 20$ & $40 \% \pm 10$ & 0.001 \\
\hline Obtuse marginal artery $(\mathrm{OM})$ & $80 \% \pm 10$ & $50 \% \pm 10$ & 0.001 \\
\hline Right coronary artery (RCA) & $70 \% \pm 30$ & $40 \% \pm 10$ & 0.001 \\
\hline
\end{tabular}

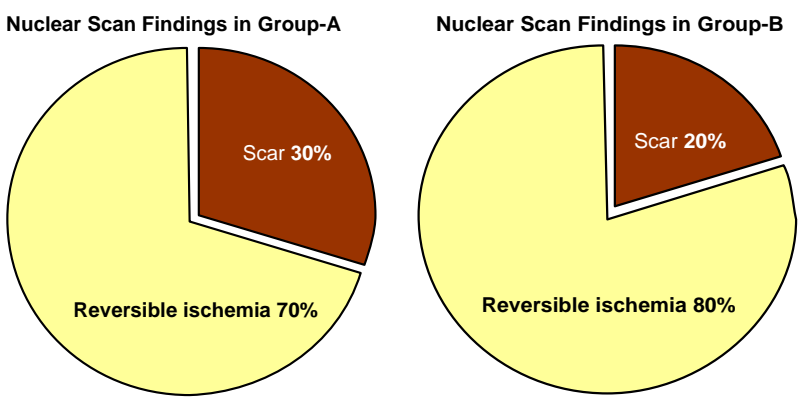

Figure 5. Showed percentages of scar tissue and reversible ischemia in both groups.

dence of MACE in group-A in the form of 6 cardiac deaths in group-A versus no cardiac deaths in group-B, Re-infarction in 8 patients in group-A versus 2 patient in group-B, symptomatic heart failure in 26 patient in group-A 5 cases from them needed a circulatory support in relation to only 8 cases developed heart failure in group-B, none of them needed a circulatory support. Cerebrovascular stroke was found in 4 cases in group-A versus no cases in group-B, sustained VT or VF needed cardioversion occurred in 18 patient in group-A versus 6 cases in group-B most of them was self-terminated. As shown in the Figure 6.

\subsection{Discussion}

Presence of fragmented QRS represents distortion of signal conduction and depolarization process within the ventricles which is related to myocardial scar/myocardial ischemia or myocardial fibrosis. The short-term and inhospital prognosis is still in dispute [26].

About the methodology used, different questions related to the technique of ECG used are raised. Das et al. in 2008 proved that, ECG recording used to detect fQRS is not a specific setting and is the same as routine 12-lead ECG recording: high-pass filter: $0.05-20 \mathrm{~Hz}$ (usually $0.15 \mathrm{~Hz}$ ), low-pass filter: $100-150 \mathrm{~Hz}$, AC filter: 50 or $60 \mathrm{~Hz}$, paper speed: 25 - $50 \mathrm{~mm} / \mathrm{sec}$ (usually $25 \mathrm{~mm} / \mathrm{sec}$ ) and voltage $1 \mathrm{~mm} / \mathrm{mV}$ [16].

Our study detected a high percentage $(30 \%)$ of irreversible scar tissues in group-A (fQRS) and this is supported by many studies where Myocardial single photon emission tomography (SPECT) was used to identify regional perfusion abnormalities from a scar by a prior myocardial infarction and significantly greater perfusion and functional abnormalities than the Q wave was proved [5, 16]. Also, the combination of $Q$ wave and fQRS improved the sensitivity $(91.4 \%)$, specificity $(89 \%)$, and negative predictable value $(92.4 \%)$ for detection of scared myocardium [27].

Wang et al. in 2010 failed to show significance for fQRS in detection of myocardial scar in patients with CAD. The reason for the different results from previous 


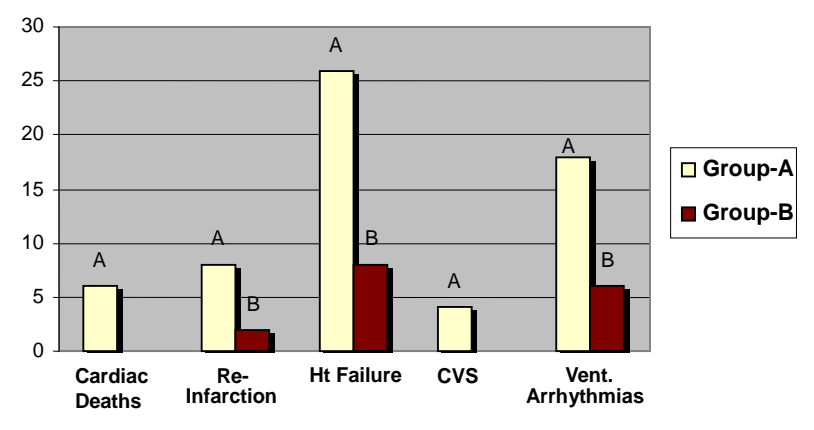

Figure 6. MACE in group-A versus group-B.

studies is unclear, and the authors stated that larger study is required to confirm the diagnostic value of fQRS [28].

Our study correlates fQRS to the coronary angiographic findings and detects a higher percent and severer lesions in fQRS-group than Non fQRS patients. The findings of Yutaka et al. in 2012, support this idea, they found that fQRS in acute coronary syndrome appeared within 48 hours (especially within 24 hours) from the onset of symptoms and persisted thereafter. fQRS on 12-lead ECG developed in $55 \%$ of patients with ST elevation myocardial infarction (STEMI) and in 50\% of patients with non-ST elevation myocardial infarction (NSTEMI), but in only $3.7 \%$ of patients with unstable angina pectoris.

Although the sensitivities of fQRS for STEMI and NSTEMI were $55 \%$ and $50 \%$ respectively, the specificity of fQRS for AMI was 96\% [29].

Our study proved that, patients with fQRS showed a lower LV functions in the form of significant increased $\mathrm{Lv}$ volumes, lower EF\% and higher MR degree than nonfQRS group. Yan et al. in 2012 demonstrated a strong relation between fragmentation of QRS and depression of LV functions even in a sub-clinical situation [30]. Also, many studies support a high sensitivity of fQRS to the presence of symptomatic LV failure in CAD patients $[8$, $31]$.

Since fQRS represents myocardial scar, fQRS may be associated with heart failure and ventricular tachyarrhythmia. Major adverse cardiac events (MACE) were studied in our research and revealed a significant $(p<0.05)$ higher incidence of cardiac deaths in group-A than groupB. Some studies have shown a strong relationship between existence of fQRS in patients with CAD and prognosis. In a study made by Das et al. 2007, patients with narrow QRS complexes that showed a fragmentation of QRS have higher all-cause mortality $(34.1 \%$ vs. $25.9 \%)$ and cardiac event rate $(49.5 \%$ vs. $27.6 \%)$ than patients without fQRS [6].

A study made by Korhonen et al. 2010, in which fQRS in patients with prior myocardial infarction was evaluated by a body surface mapping system also showed that fQRS was an independent predictor for cardiac death and hospitalization due to heart failure [31].
Torigoe et al. 2012 evaluated fQRS by 12-lead ECG and showed that the number of the leads with fQRS was a predictor for cardiac death and hospitalization for heart failure in patients with prior myocardial infarction. Although prior criteria for positive fQRS included the presence of fQRS in 2 or more contiguous leads, they showed that the presence of fQRS in 3 or more leads was the most useful for distinguishing between patients with and without risk for cardiac death or hospitalization [32].

An increase in the number of leads with fQRS would represent a wide scar area, which would result in an adverse outcome. fQRS also predicted intra- and post-operative hemodynamic instabilities and adverse cardiovascular events in patients undergoing coronary artery bypass graft surgery. Mortality of patients with f-wQRS was significantly higher than that of patients without $\mathrm{f}$ wQRS, and subgroups of f-wQRS (fragmented left BBB, fragmented PVC and fragmented paced QRS) were also associated with short time to death compared to patients without $\mathrm{f}-\mathrm{wQRS}$. Multivariate analysis showed that reduced EF $(\leq 35 \%)$ and f-wQRS were independently associated with mortality [33].

For more confirmation of the effect of fragmentation of QRS in ECG, a number of follow-up studies [32,34] discovered a strong relation between fragmentation of QRS and cardiac death. The findings of Pietrasik et al., in 2010, who studied the appearance of fQRS in ECG of patients with first Q-wave MI, resolved Q wave, and persistent Q wave on 2 month follow-up and its relation to the risk of recurrent adverse cardiac events defined as, unstable angina, recurrent MI or cardiac death. They found a significant correlation between fQRS with the higher risk of recurrent adverse cardiac events among patients with resolved Q wave. It was proposed that fQRS may identify ischemic myocardium [34].

Another research found a strong correlation between fQRS and cardiac mortality in long-tem periods especially in those need ICD or CRT therapy. Das et al. studied 361 patients in a follow-up period from 6 months to more than 2 years and detected a significant increased risk of arrhythmic events but no effect on mortality [17]. Brenyo et al. [35] and Pietrasik et al. [34] during (19 \pm 12 months) discover no effect in total population; however, increased risk of death only among patients with LBBB and inferior fQRS.

Cheema et al. evaluated the association between fQRS complex and primary end-point of all cause mortality and secondary end-points specific cause mortality and appropriate ICD shocks in the cohort of 842 patients with LV dysfunction (LVEF $\leq 35 \%$ ), both ischemic and non-ischemic etiology [8].

Fragmented QRS was present in $32.5 \%$ of patients. Mean follow-up of the study was $40 \pm 17$ months for entire cohort. There was no significant difference in event- 
free survival between patients with $\mathrm{fQRS}$ and without fQRS. Fragmented QRS was not associated with increased risk of all-cause mortality, arrhythmic mortality and non-arrhythmic mortality. Similar conclusions were drawn from studies by Forleo et al. [36].

\subsection{Limitations of the Study}

1) fQRS on a 12-lead ECG requires an optimal low pass filter setting $(100$ or $150 \mathrm{~Hz}$ ). Fragmentation may be missed with a filter setting of 40 or $60 \mathrm{~Hz}$. Apart from notching, slurring of fQRS may also represent myocardial scar.

2) The present definition of fQRS is being qualitative, preventing the incorporation of slurring in this phenomenon. A quantitative definition of fQRS with development of specific software is underway.

3) It is to be emphasized that fQRS is a nonspecific finding and should only be interpreted in the presence of pertinent clinical evidence of myocardial scar as in CAD or primary electrical abnormalities of depolarization such as Brugada syndrome.

4) The effects of different therapeutic strategies (thrombolysis or revascularization) on fQRS and prognosis of CAD patients need further studies.

5) Correlation between QRS fragmentation and the novel selvester QRS-score has to be established.

6) A further follow-up should be evaluated well as many researches did.

7) A larger population sampling supports such tools to increase its validity.

\section{Acknowledgements}

To the team work colleagues in Non-invasive unit of Cardiology Dept. El Minia University Hospital and the secretary employees of the department for easy call back and archiving process.

\section{REFERENCES}

[1] N. C. Flowers, L. G. Horan, J. R. Thomas and W. J. Tolleson, "The Anatomic Basis for High-Frequency Components in the Electrocardiogram," Circulation, Vol. 39, No. 4, 1969, pp. 531-539. doi:10.1161/01.CIR.39.4.531

[2] P. L. Friedman, J. J. Fenoglio and A. L. Wit, "Time Course for Reversal of Electrophysiological and Ultrastructural Abnormalities in Subendocardial Purkinje Fibers Surviving Extensive Myocardial Infarction in Dogs," Circulation Research, Vol. 36, No. 1, 1975, pp. 127-144. doi:10.1161/01.RES.36.1.127

[3] P. Varriale and B. E. Chryssos, "The RSR' Complex Not Related to Right Bundle Branch Block: Diagnostic Value as a Sign of Myocardial Infarction Scar," American Heart Journal, Vol. 123, No. 2, 1992, pp. 369-376. doi:10.1016/0002-8703(92)90648-F
[4] A. Bayes de Luna, "Electrographic Patterns of Ischemia, Injury and Necrosis," In: A. Bayes de Luna, Ed., Clinical Electrocardiography: A Textbook, 2nd Edition, Futura Publishing Company, Inc., Armonk, 1998, pp. 141-142.

[5] M. K. Das, B. Khan, S. Jacob, A. Kumar and J. Mahenthiran, "Significance of a Fragmented QRS Complex Versus a Q Wave in Patients with Coronary Artery Disease," Circulation, Vol. 113, No. 21, 2006, pp. 2495-2501. doi:10.1161/CIRCULATIONAHA.105.595892

[6] M. K. Das, C. Saha, H. El Masry, et al., "Fragmented QRS on a 12-Lead ECG: A Predictor of Mortality and Cardiac Events in Patients with Coronary Artery Disease," Heart Rhythm, Vol. 4, No. 11, 2007, pp. 13851392. doi:10.1016/j.hrthm.2007.06.024

[7] M. Yuce, V. Davutoglu, B. Ozbala, et al., "Fragmented QRS Is Predictive of Myocardial Dysfunction, Pulmonary Hypertension and Severity in Mitral Stenosis," The Tohoku Journal of Experimental Medicine, Vol. 220, 2010, pp. 279-283. doi:10.1620/tjem.220.279

[8] A. Cheema, A. Khalid, A. Wimmer, et al., "Fragmented QRS and Mortality Risk in Patients with Left Ventricular Dysfunction," Circulation: Arrhythmia and Electrophysiology, Vol. 3, 2010, pp. 339-344. doi:10.1161/CIRCEP.110.940478

[9] G. Breithardt, M. E. Cain, N. el-Sherif, et al., "Standards for Analysis of Ventricular Late Potentials Using HighResolution or Signal-Averaged Electrocardiography. A Statement by a Task Force Committee of the European Society of Cardiology, the American Heart Association, and the American College of Cardiology," Circulation, Vol. 83, No. 4, 1991, pp. 1481-1488. doi:10.1161/01.CIR.83.4.1481

[10] J. A. Gomes, M. E. Cain, A. E. Buxton, M. E. Josephson, K. L. Lee and G. E. Hafley, "Prediction of Long Term Outcomes by Signal-Averaged Electrocardiography in Patients with Unsustained Ventricular Tachycardia, Coronary Artery Disease, and Left Ventricular Dysfunction," Circulation, Vol. 104, 2001, pp. 436-441. doi:10.1161/hc2901.093197

[11] H. Morita, K. F. Kusano, D. Miura, et al., "Fragmented QRS as a Marker of Conduction Abnormality and a Predictor of Prognosis of Brugada Syndrome," Circulation, Vol. 118, No. 17, 2008, pp. 1697-1704. doi:10.1161/CIRCULATIONAHA.108.770917

[12] M. K. Das, H. Suradi, W. Maskoun, M. A. Michael, C. Shen, J. Peng, et al., "Fragmented Wide QRS on a 12Lead ECG: A Sign of Myocardial Scar and Poor Prognosis," Circulation: Arrhythmia and Electrophysiology, Vol. 1, 2008, pp. 258-268. doi:10.1161/CIRCEP.107.763284

[13] P. I. Gardner, P. C. Ursell, J. J. Fenoglio Jr. and A. L. Wit, "Electrophysiologic and Anatomic Basis for Fractionated Electrograms Recorded from Healed Myocardial Infarcts," Circulation, Vol. 72, No. 3, 1985, pp. 596-611. doi:10.1161/01.CIR.72.3.596

[14] I. Wiener, B. Mindich and R. Pitchon, "Determinants of Ventricular Tachycardia in Patients with Ventricular Aneurysms: Results of Intraoperative Epicardial and Endocardial Mapping," Circulation, Vol. 65, 1982, pp. 856861. doi:10.1161/01.CIR.65.5.856 
[15] I. Wiener, B. Mindich and R. Pitchon, "Fragmented Endocardial Electrical Activity in Patients with Ventricular Tachycardia: A New Guide to Surgical Therapy," American Heart Journal, Vol. 107, 1984, pp. 86-90. doi:10.1016/0002-8703(84)90138-8

[16] M. K. Das, H. Suradi, W. Maskoun, et al., "Fragmented wide QRS on a 12-Lead ECG: A Sign of Myocardial Scar and Poor Prognosis," Circulation: Arrhythmia and Electrophysiology, Vol. 1, No. 4, 2008, pp. 258-268. doi:10.1161/CIRCEP.107.763284

[17] M. K. Das, W. Maskoun, C. Shen, M. A. Michael, H. Suradi, M. Desai, et al., "Fragmented QRS on TwelveLead Electrocardiogram Predicts Arrhythmic Events in Patients with Ischemic and Nonischemic Cardiomyopathy," Heart Rhythm, Vol. 7, No. 1, 2010, pp. 74-80. doi:10.1016/j.hrthm.2009.09.065

[18] M. Homsi, L. Alsayed, B. Safadi, J. Mahenthiran and M. K. Das, "Fragmented QRS Complexes on 12-Lead ECG: A Marker of Cardiac Sarcoidosis as Detected by Gadolinium Cardiac Magnetic Resonance Imaging," Annals of Noninvasive Electrocardiology, Vol. 14, No. 4, 2009, pp. 319-326. doi:10.1111/j.1542-474X.2009.00320.x

[19] S. Peters, M. Trümmel and B. Koehler, "QRS Fragmentation in Standard ECG as a Diagnostic Marker of Arrhythmogenic Right Ventricular Dysplasia-Cardiomyopathy," Heart Rhythm, Vol. 5, No. 10, 2008, pp. 1417-1421. doi:10.1016/j.hrthm.2008.07.012

[20] M. Takagi, Y. Yokoyama, K. Aonuma, N. Aihara and M. Hiraoka, "Clinical Characteristics and Risk Stratification in Symptomatic and Asymptomatic Patients with Brugada Syndrome: Multicenter Study in Japan," Journal of Cardiovascular Electrophysiology, Vol. 18, No. 12, 2007, pp. 1244-1251. doi:10.1111/j.1540-8167.2007.00971.x

[21] S. G. Priori, M. Gasparini, C. Napolitano, et al., "Risk Stratification in Brugada Syndrome: Results of the Prelude (Programmed ELectrical Stimulation Predictive Value) Registry," Journal of the American College of Cardiology, Vol. 59, No. 1, 2012, pp. 37-45. doi:10.1016/j.jacc.2011.08.064

[22] C. Antzelevitch, "Role of Transmural Dispersion of Repolarization in the Genesis of Druginduced Torsades de Pointes," Heart Rhythm, Vol. 2, No. 11, 2005, pp. S9S15. doi:10.1016/j.hrthm.2004.09.011

[23] K. Haraoka, H. Morita, Y. Saito, et al., "Fragmented QRS Is Associated with Torsades de Pointes in Patients with Acquired Long QT Syndrome," Heart Rhythm, Vol. 7, No. 12, 2010, pp. 1808-1814. doi:10.1016/j.hrthm.2010.09.008

[24] S. J. Park, Y. K. On, J. S. Kim, et al., "Relation of Fragmented QRS Complex to Right Ventricular Fibrosis Detected by Late Gadolinium Enhancement Cardiac Magnetic Resonance in Adults with Repaired Tetralogy of Fallot," American Journal of Cardiology, Vol. 109, No. 1, 2012, pp. 110-115. doi:10.1016/j.amjcard.2011.07.070

[25] H. Kadi, A. Inanir, A. Habiboglu, et al., "Frequency of Fragmented QRS on ECG Is Increased in Patients with Rheumatoid Arthritis without Cardiovascular Disease: A
Pilot Study," Modern Rheumatology, 2011. doi:10.1007/s10165-011-0493-9

[26] G. Pietrasik and W. Zareba, "QRS Fragmentation: Diagnostic and Prognostic Significance," Cardiology Journal, Vol. 19, No. 2, 2012, pp. 114-121. doi:10.5603/CJ.2012.0022

[27] C. V. Reddy, K. Cheriparambill, B. Saul, et al., "Fragmented Left Sided QRS in Absence of Bundle Branch Block: Sign of Left Ventricular Aneurysm," Annals of Noninvasive Electrocardiology, Vol. 11, 2006, pp. 132138.

[28] D. D. Wang, D. M. Buerkel, J. R. Corbett and H. S. Gurm, "Fragmented QRS Complex Has Poor Sensitivity in Detecting Myocardial Scar," Annals of Noninvasive Electrocardiology, Vol. 15, No. 4, 2010, pp. 308-314. doi:10.1111/j.1542-474X.2010.00385.x

[29] Y. Take and H. Morita, Indian Pacing and Electrophysiology Journal, Vol. 12, No. 5, 2012, pp. 213-225.

[30] G. H. Yan, M. Wang, K. H. Yiu, et al., "Subclinical Left Ventricular Dysfunction Revealed by Circumferential 2D Strain Imaging in Patients with Coronary Artery Disease and Fragmented QRS Complex," Heart Rhythm, Vol. 9, No. 6, 2012, pp. 928-935. doi:10.1016/j.hrthm.2012.01.007

[31] P. Korhonen, T. Husa, T. Konttila, et al., "Fragmented QRS in Prediction of Cardiac Deaths and Heart Failure Hospitalizations after Myocardial Infarction," Annals of Noninvasive Electrocardiology, Vol. 15, No. 2, 2010, pp. 130-137. doi:10.1111/j.1542-474X.2010.00353.x

[32] K. Torigoe, A. Tamura, Y. Kawano, K. Shinozaki, M. Kotoku and J. Kadota, "The Number of Leads with Fragmented QRS Is Independently Associated with Cardiac Death or Hospitalization for Heart Failure in Patients with Prior Myocardial Infarction," Journal of Cardiology, Vol. 59, No. 2, 2012, pp. 36-41.

doi:10.1016/j.jjcc.2011.09.003

[33] T. Erdogan, M. Cetin, S. A. Kocaman, et al., "Relationship of Fragmented QRS with Prognostic Markers and In-Hospital MACE in Patients Undergoing CABG," Scandinavian Cardiovascular Journal, Vol. 46, No. 2, 2012, pp. 107-113. doi:10.3109/14017431.2011.651485

[34] G. M. Pietrasik, S. Polonsky, A. J. Moss and W. Zareba, "Presence of Fragmented Wide-QRS Complex and the Risk of Death and Sudden Cardiac Death among MADIT-II Patients with Left Bundle Branch Block," ACC Poster, Atlanta, 2010.

[35] A. J. Brenyo, G. Pietrasik, S. McNitt and W. Zareba, "QRS Fragmentation: Lack of Association with Cardiac Events in Patients with a Normal QRS Duration," Denver, Co., 2010.

[36] G. B. Forleo, D. G. Della Rocca, L. P. Papavasileiou, et al., "Predictive Value of Fragmented QRS in Primary Prevention Implantable Cardioverter Defibrillator Recipients with Left Ventricular Dysfunction," Journal of Cardiovascular Medicine (Hagerstown), Vol. 12, No. 11, 2011, pp. 779-784. doi:10.2459/JCM.0b013e32834ae458 


\section{List of Abbreviations}

EF: Ejection Fraction

NYHA: New York Health Association Classification

MACE: Major Adverse Cardiac Events

ACS: Acute Coronary Syndrome
fQRS: Fragmented QRS-Complex in ECG ECG: Electrocardiography GE: General Electric

ICD: Intracardiac Defibrillation Device CRT: Cardiac Re-Synchronization Therapy 